\title{
A New Plant Cell Image Segmentation Algorithm ${ }^{\star}$
}

\author{
G. Fernàndez ${ }^{1}$ and M. Kunt ${ }^{1}$ and J-P. Zrÿd $\mathrm{d}^{2}$ \\ 1 Signal Processing Laboratory \\ Swiss Federal Institute of Technology \\ CH-1015 Lausanne, Switzerland \\ 2 Laboratory of Plant Cell Genetics \\ University of Lausanne \\ CH-1015 Lausanne, Switzerland
}

\begin{abstract}
A new plant cell image segmentation algorithm is presented in this paper. It is based on the morphological analysis of the cell shapes and on gradient information. The difficulty of the segmentation of plant cells lies in the complex shapes of the cells and their overlapping, often present due to recent cellular division. The algorithm presented tries to imitate the human procedure for segmenting overlapping and touching particles. It analyzes concavities in the shape of a group of cells as well as the existence of a coherent surface for the segmentation. The algorithm can be divided in two main parts, firstly a simple method for finding dominant concave points in shapes is introduced and secondly several parameters between concave points are calculated as a criterion for segmentation. It has been also shown that the algorithm produces good results when the output is applied as a marker for the morphological watershed algorithm. Results will be presented in real images of a cell suspension culture to show the validity of the chosen approach.
\end{abstract}

\section{Introduction}

In biology, a common problem is the segmentation of cells for counting and feature extraction purposes. Manual work is tedious and yields imprecise and subjective results. Computer assisted assessment has been shown to be a powerful tool due to its automatic, objective and fast measurement. A typical biological image analysis system starts with a digitizer (a video camera, scanner, etc..) followed by an enhancement of the image, segmentation of the cells and feature extraction for classification. The output provides statistical results concerning the state of the culture that can be interpreted by the biologists.

Image segmentation has been studied by many authors and it has been shown that it is often dependent on the type of elements to segment. A relatively new tool for segmentation is the watershed algorithm [1]. It has the ability to perform very accurate segmentations from image markers reducing the problem

\footnotetext{
* This work is supported by Swiss National Science Foundation (FNRS) grant N.
} 
to a simple marker extraction. In this paper we present a new method to find markers of touching and overlapping cells that gives good results in combination with the watershed algorithm.

\section{$2 \quad$ Project Background}

Samples of Beta vulgaris cells are cultivated in vitro in liquid suspension. Previous work in this project yielded well enhanced images. Samples of the cells are conveniently diluted in order to have a reasonable spatial distribution. After digitation of the cells [2], the useful zone of the scene was obtained by an adaptive form of the Hough transformation [3] and the images were enhanced by the morphological top hat transformation [4]. The next step in the image analyzing process is the segmentation of the enhanced cells and will be discussed in the next sections.

\section{Segmentation of Plant Cells}

The enhanced cells present an even spatial distribution and some of them are touching or overlapping. They do not present a unique geometrical shape that could facilitate the marker extraction (i.e. ultimate erosion yields oversegmentation) the grey level is similar for all of them (the gradient information is often useless). Furthermore, the gradient image does not present a unique minimum per cell which could be used for the marker extraction. It is for all these reasons that classical segmentation algorithms either do not segment the touching cells or lead to oversegmentation. The new segmentation method presented here tries to solve this problem.

The main idea of the algorithm lies in the imitation of the human procedure in segmenting touching particles. A group of cells is segmented if it presents a shape with two concavities relatively near one to each other with respect to the perimeter of the group, and if a flat path exists between the concave points.

\subsection{Contours and Their Concavities}

After the enhancement by the top hat transformation, groups of cells are clearly differentiated from the background. The next step is to obtain the contours of the cells in order to study their concavities. This is done by the set difference between the binary image and the unitary erosion. The contours are extracted, labeled and analyzed to obtain the concave points.

\subsection{Concave Points}

Dominant points have been widely studied in Pattern Recognition literature, specially in polygonal approximation purposes, object and character recognition but not so much in segmentation $[5,6]$. These algorithms are usually recursive 
and/or computationally demanding. Since the analysis of these points must be done for all the cells in the image and the number of cells can be well above several hundreds, the algorithm of concave points has to be simple and robust.

Each point of the contour will have a value of concaveness which is calculated in the following way:

$$
\text { concaveness }(j)=\sum_{j-1}^{j+1} \sum_{x=1}^{5} \sum_{y=1}^{5} M_{j} \cap B
$$

where $B$ is the binary image, $j$ runs over the contour and $M_{j}$ is a $5 \times 5$ mask. In other words, for the $j_{t h}$ point of the contour the value of concaveness is calculated as the number of points of a $5 \times 5$ mask centered on $j$ that intersect the binary shape. Finally, in order to avoid uncertainties, the values of the two adjacent contour neighbors are added to the present contour value. Thresholding of these values yields the concave points of the shape. If two adjacent points are selected, the maximum of them is retained. Fig. 1 illustrates the method.

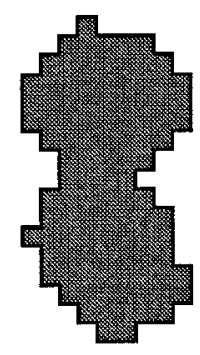

a

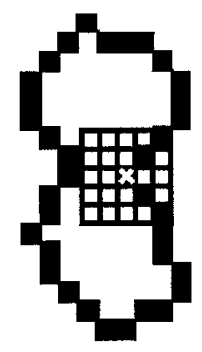

b

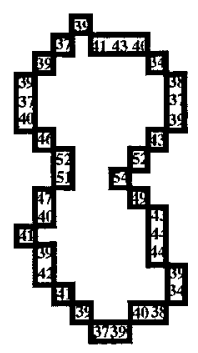

c

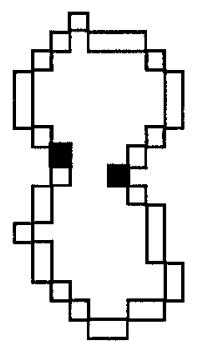

d

Fig. 1. a. Binary shape, b. Contour obtained by erosion and mask, c. Values of concaveness. d. Final concave points

These points can be due to the specific shape of the cells or the presence of touching or overlapping cells. In the next section the information of these concavities will be discussed to perform the segmentation.

\subsection{Linking Concave Points for Segmentation}

The main idea for segmenting is to cut the shapes by the places where the concave points are present. But the problem is that some concave points may be due to the particular features of the cells and, furthermore, the presence of more than two cells implies several pairs of concave points. The choice of the points for segmentation are based on two main features: The gray level information 
(flatness of the path between points) and spatial information (distance between points compared to the perimeter of the contour).

For each concave point the length and the gradient of the path to the other concave points is calculated. According to these parameters, each point selects its candidate as a partner. A concave point will choose the partner that minimizes the spatial and gradient parameters. Their description follows.

Spatial Parameter. The spatial parameter is the euclidean distance between two points. In order to prevent from segmenting cells that have concave points in their "real" shape, a condition has to be imposed: The euclidean distance between candidate segmenting points is compared to the perimeter of the shape (number of contour points) in the following way:

$$
d<\frac{p e r}{\pi}
$$

where $d$ is the distance between points and per the perimeter. The idea of the condition is to segment only when the distance $d$ is smaller than the diameter of a hypothetic circumference of perimeter per.

Gradient Parameter. If the concave points are real segmenting points, a relatively "flat path" must exist between them. The following parameter is obtained between each concave point:

$$
\operatorname{grad}=\sum_{n=1}^{i}\left|Y_{n}-Y_{n-1}\right|
$$

where $i$ is the number of points in the line that joins two concave points and $Y_{n}$ the gray level information in the point $n$. The point that minimizes this factor is chosen as a candidate. The obtained value must not exceed $2 * N(N$ being the number of total grey levels), which has been found to be a reasonable threshold of flatness.

The calculation of these parameters is done for all points and each point makes its choice. Finally, the selected points are compared, when the points select one to each other the cell is decided to be segmented by drawing a line between points. This line is dilated in order to let the watershed algorithm to find a more accurate segmentation line. In Fig. 2 where the algorithm is illustrated, each point selects its partner but only the mutual choice produces segmentation.

\section{Results}

For testing the performance of the algorithm, a collage image was built containing groups of cells (Fig. 3.a). Different stages of the algorithm are shown, contour image with concave points, segmenting lines and final segmented image after the watershed algorithm. 

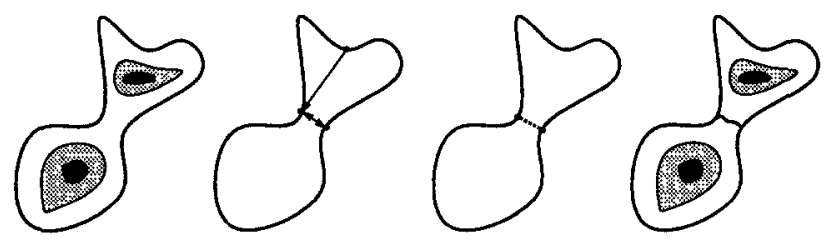

Fig. 2. Each concave point selects its candidate but only when a mutual choice occurs the segmentation is done.

The algorithm performs good segmentation in trivial cases where the shape has a pair of clear concavities close one to the other. For more complex combinations of cells, the results are also satisfactory, taking into account that sometimes even for the biologist it is difficult to say how many cells are in a group. It is important to remark the good selection of linking the points in the cases where even if they are fare one to each other, the algorithm recognizes them as the right path to chose.

After the segmentation of the cells, features such as length, width, and area are calculated for a final classification.

\section{Conclusions}

In this paper, a new plant cell image segmentation algorithm has been presented. It is based on the imitation of the human segmentation, that means segmentation of cells between the points that copes certain features of shape and surface within the cell. The algorithm has been shown to be robust in front of different cell shapes and groups of cells. Results in cultures in liquid suspension satisfy the segmentation needs for a complete image analysis system for plant cells.

\section{Acknowledgments}

The authors would like to thanks Mr P.Brigger for his useful suggestions in this work.

\section{References}

1. S. Beucher and F. Meyer. Morphological segmentation. Journal of Visual Communication and Image Representation, 1:21-46, 1990.

2. P. Brigger. Morphological plant cell analysis. In J. Serra and P. Salembier, editors, Mathematical Morphology and its Applications to Signal Processing, pages 101-106, Barcelona, May 1993. 


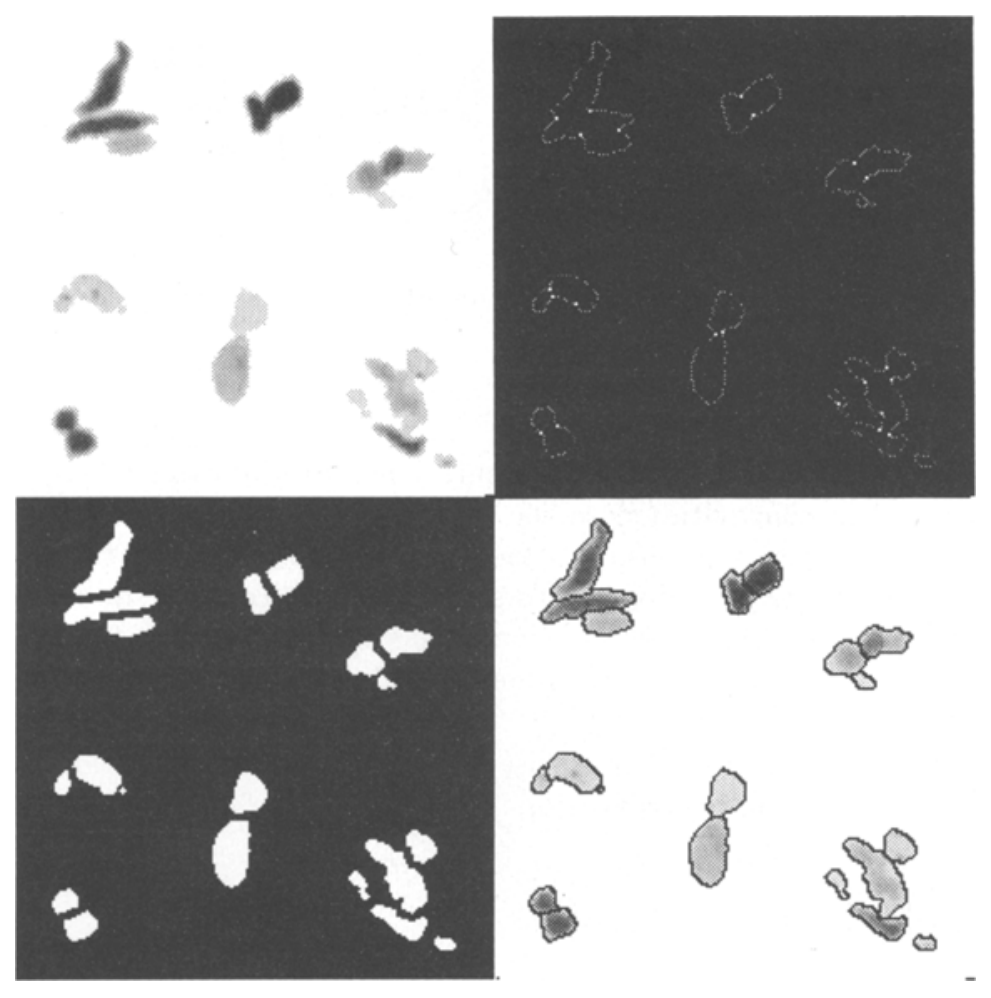

Fig. 3. From left to right and top to bottom: a. Original collage image, b. concave points of contours, c. segmenting lines, d. final segmented image after the watershed algorithm.

3. R. Duda and P. Hart. Use of the hough transformation to detect lines and curves in pictures. Communications of the ACM, 15:11-15, 1972.

4. F. Meyer. Cytologie quantitative et morphologie mathématique. PhD thesis, Ecole Nationale Supérieure des Mines de Paris, Fontainebleau, 1979.

5. A.K. Jain, S.P. Smith, and E. Backer. Segmentation of muscle cell pictures: a preliminary study. IEEE Transactions on Pattern Analysis and Machine Intelligence, $3: 232-242,1980$.

6. A. Lerch and D. Chetverikov. Correction of line drawings for image segmentation in leather industry. In Proc. 11th Int. Conf. on Pattern Recognition, volume 1, pages 420-424, The Hague, 1992. 